\title{
Nanotechnology in Oral Cancer Prevention and Therapeutics: A Literature Review
}

\author{
Ritwika Kumar ${ }^{1}$ Kunal Jha² Diplina Barman ${ }^{3}$ \\ 1School of Materials Science and Nano Technology, Jadavpur \\ University, Kolkata, West Bengal, India \\ ${ }^{2}$ Department of Public Health Dentistry, Kalinga Institute of Dental \\ Sciences, KIIT University, BBSR, Bhubaneswar, Odisha, India \\ ${ }^{3}$ Private Dental Practitioner, Hooghly, West Bengal, India \\ Ind J Med Paediatr Oncol 2021;42:146-152.
}

\author{
Address for correspondence Diplina Barman, MDS, Public Health \\ Dentistry Private Dental Practitioner, 23, P.C. Seal Road, \\ P.O. Chandannagar, Dist, Hooghly 712136, India \\ (e-mail: diplina93@yahoo.co.in).
}

\section{Introduction}

Since time immemorial, the word "nano" has improved its applications and has made way into the world's daily performances. It has fabricated assumption about the tremulous shift in various aspects of engineering and medical sciences. This is a Greek word meaning "dwarf." A nanometer $(\mathrm{nm})$ measures one thousand millionth of a meter. ${ }^{1}$ The American Chemical Society defined nanotechnology as the design (at the atomic, molecule, and supra molecular levels) characterization, production, and utilization of constitutions, equipment, and systems by controlling shape and size at a nanometer scale. ${ }^{2}$

\section{Types of Nanoparticles}

$>$ Nanosuspensions are colloidal dispersions of nano-sized drug particles that are produced and synthesized by a suitable stabilizer. The size ranges from 30 to $100 \mathrm{~nm}$.

$>$ Nanoparticles (NPs) are of two types namely, nanospheres and nanocapsules.

- Nanospheres are polymer matrices in which the drug is dissolved and dispersed.

- Nanocapsules consist of polymer walls entrapping oil in the core where the drug is diffused. They have advantages of improved efficiency, reduction in toxicity levels, improved distribution, and observance. ${ }^{3}$

\footnotetext{
(C) 2021. Indian Society of Medical and Paediatric Oncology.

This is an open access article published by Thieme under the terms of the Creative Commons Attribution-NonDerivative-NonCommercial-License, permitting copying and reproduction so long as the original work is given appropriate credit. Contents may not be used for commercial purposes, or adapted, remixed, transformed or built upon. (https://creativecommons.org/licenses/by-nc-nd/4.0/).

Thieme Medical and Scientific Publishers Private Ltd. A-12, Second Floor, Sector -2, NOIDA -201301, India
} 
The different categories of NP include polymeric, solid lipid, magnetic, nanopores, quantum dots, polymeric micelles, ceramic NPs, nanowires, liposomes, dendrimers, nanoshells coated with gold, and ferrofluids.

NPs operate on innate immune systems and activate anticancer immune reaction. Innate immunity helps in maintaining the integrity of the body, by detecting and eliminating damaged cells, proteins, and immediate defense against foreign invading agents. Interaction of NPs with monocytes and macrophages has been widely studied in a range of cellular models in vitro and in vivo experimental animals. This depends on the route of entry into the body and on the consequent "coating" of the reactive NP surface with microenvironmental proteins. The ability of NPs to adsorb different types of molecules in a particular tissue microenvironment makes a huge difference in innate immunity to recognize them as foreign entities and to mount an inflammatory response. ${ }^{4,5}$

\section{Properties of Nanoparticles}

Three layers are present in the NPs: the core, the surface layer, and the shell layer. Surface layer consists of metal ions, surfactants, and polymers. There are metallic NPs, nonmetal, ceramic, lipid, polymeric, and semiconductor NPs. The properties vary according to the available sizes and type of material. ${ }^{6}$

Super hard NPs are those which are sized below $50 \mathrm{~nm}$. The malleability and ductility also vary. Magnetic materials show super para magnetism properties in some while quantum confinement Q-particles and surface plasmon resonance in some. Some show solar radiation in photovoltaic cells and enhanced diffusion at elevated temperatures owing to high surface area to volume ratio. Improved image resolution and contrast is another property of NPs because the NPs at approximating infrared wavelengths initiate localized surface plasmon resonances. ${ }^{7}$

\section{Applications of Nanotechnology}

NPs are used in targeted drug delivery and the controlled release of curative agents and the entire system of nanotechnology is an engineered technology with multiple applications. Recent and newer materials could be fabricated to impersonate the human bone crystal mineral structure and could also be used as dental restorative resin. Nanomaterials have been used in dentifrices and mouth rinses for improved oral healthcare. They are also used as dental adhesives, anticariogenic enamel polishing agent, and implant materials much more successfully than the conventional materials. They act as antimicrobial agents by preventing bacterial proliferation. Various nanosized carriers are efficiently involved in improving pharmacokinetics and accumulation reduces the adverse effect of antibiotics. The NPs are retained in our body for a longer time for achieving sustained therapeutic effects. ${ }^{8}$ Commercial applications have tailored gold NPs as tools to detect targeted sequences of nucleic acids that are clinically scrutinized as potential curatives for malignant conditions along with other diseases ${ }^{9}$ and to diagnose and follow up oral malignant conditions. ${ }^{10}$

\section{Advantages of Nanotechnology}

> Cost-effectiveness, appropriate time management, reduced patient anxiety as it is noninvasive.

$>$ Biocompatible, easily synthesized, and has the potential to reach specific target molecules.

$>$ Reduction in drug resistance, tumor recurrence, and metastasis prevention.

With increased dependency on the evidence-based-medicine, reviewing literature pertaining to diagnostic and therapeutic improvements in the application of nanotechnology is gaining importance. Thus, the aim of this literature review is to critically appraise the various researches that have been reported around the globe over the past few decades. 3,11

\section{Disadvantage of Nanotechnology}

There are certain health effects of NPs that should be considered. Various levels of toxicity have been reported for various nanomaterials. Toxicity of ferric NPs has been reported. There are reports showing prevalence of cardiovascular disease with the increased presence of particular matter in the air. Some of the NPs have been reported to cross blood-brain barrier, thereby challenging its application in the field of drug delivery. ${ }^{11}$

\section{Background}

Maxillofacial malignancies (oral cavity and oropharynx) are the sixth most common type of cancer globally. It invades local tissue, metastasizes, and has a high mortality rate. Although there have been improvements in the medical therapeutics, not much improvement in the survival rate has been observed over the past two decades. Oral cancer is often diagnosed at progressed stages when the malignant cells become aggressive and immune to curative medications. In India, five lives are lost hourly to oral cancer. The prevalence is higher in males as compared with females. Oral cancer incidence is 8.4 times higher in patients with smoking and tobacco chewing habits. ${ }^{12}$

Preventive measures are on a rise to restrict the increasing incidence and mortality of oral cancer. The prognosis of malignant lesions is dependent on the time of detection. Use of nanodetection systems is increasing, and has emerged as potential noninvasive systems bringing the detection sensitivity of biomarkers to nanoscale. ${ }^{13}$ Early diagnosis of potentially malignant oral disorders like leucoplakia, discoid lupus erythematosus, erythroplakia, actinic keratosis, oral lichen planus, and oral submucous fibrosis would help in improving the prognosis of the lesion. ${ }^{14}$

Thus, recent diagnostic methods should be researched on with better clinical benefits that would accurately predict the risk of oral potentially malignant disorders, malignant 
transformation, diagnose oral cancer based on molecular targeting, provide ultrasensitive diagnosis strategies at nanoscale, created real-time markings for the extent of surgical resection margins, and evaluate oral cancer prognosis in an expedient way posttreatment.

\section{Methodology}

\section{Article Selection for the Review}

Original studies and review articles related to nanotechnology, history of development, clinical implications of nanotechnology in oral cancer prevention, and therapeutics published between 1990 and 2020 were considered for the study. A total of 200 articles were searched using various databases: SCOPUS, PUBMED, PUBMED CENTRAL, MEDLINE, and WEB OF SCIENCE database.

\section{Nanotechnology-Based Therapeutic Approaches in the Treatment of Oral Carcinoma}

1. Photodynamic therapy (PDT)

2. Stem cell therapy

3. Nanotechnology-based radiation immunotherapy

4. Ultrasound nanotheranostics

5. Drug-delivery systems based on nanotechnology in oral cancer therapy

\section{Photodynamic Therapy}

Ceron Jayme et al developed DNA polymer films (PFs), which she used as a drug delivery method for treating oral carcinoma. DNA-PFs integrated with a photoactive compound: chlorine aluminum phthalocyanine (DNA-PFs-AlClPc) were used to cram the growth prototype of oral squamous cell carcinoma (OSCC) cells. The cell viability was measured using flow cytometry post-application of DNA-PFs-AlClPc during PDT. A positive response was noted toward photostimulation with the light dosage including 300,600 , and $1200 \mathrm{~mJ} / \mathrm{cm}^{2}$. Reduced viability (30\% at the highest dose $\left[1200 \mathrm{~mJ} / \mathrm{cm}^{2}\right]$ ) and increased apoptosis were noted with escalating doses as compared with controls. Apoptosis was the main mechanism of cell death. This system recommends the application of DNA-PFs-AlClPc as a drug delivery system for active molecules in treating pathologies. This also indicates the potency of using nanostructure in novel ways to treat malignancies. DNA nanostructured films could also be made to use as a "curative material." 15

Eka-Putra et al conducted a study using PDT mixed to lipid platinum chloride NPs to inhibit the multiplication of OSCC. Lipid-platinum-chloride nanoparticles (LPC NPS) were formed by encapsulating cisplatin (CDDP) into liposomes and anticancer effects in lesions like: liver cancer, bladder and melanoma models. A xenograft model of OSCC was used to revise the therapeutic effect of PDT+LPC and a significant decrease in the lesion volume was noted ( 112\%). Minimal side effects were noted in PDT+LPC or LPC treatment. PDT+LPC protracted inhibition of the growth of tumor cells that resulted in minimal chemotherapy curatives. A potential synergistic activity of chemotherapeutic efficacy resulted. ${ }^{16}$

Zhu et al put forward a proposal where he used ferroptosis-promoted PDT based on biochemical characteristics of cellular ferroptosis, which resulted in enhanced PDT efficacy due to them by generation of reactive oxygen species and $\mathrm{O}_{2}$ feasibly via Fenton reaction. ${ }^{17}$ Xue at al developed Trojan-Horse nanoparticle (pPhD NP) that was in dual size/charge-transformable for delivering ultra-small, fully active pharmaceutical ingredients nanotheranostics with incorporated dual-modal imaging and trimodal therapeutic activities. ${ }^{18}$ Gupta et al reported the multitasking potencies of Ag-embedded $\mathrm{ZnO}$ nanocomposites (Ag-ZnO NCs). The improved photocatalytic and antibacterial activity of Ag-ZnO $\mathrm{NCs}$ is because of $\mathrm{Ag} \mathrm{NC}$ anchorage onto $\mathrm{ZnO}$ along with minor substitution of $\mathrm{Ag}$ ions in the lattice of $\mathrm{ZnO}$.

Number of studies have reported that $\mathrm{Ag}_{5}-\mathrm{ZnO} \mathrm{NCs}$ destroys oral carcinoma cells under visible light irradiation, by working as photosensitizers toward PDT of malignancy due to the outstanding photocatalytic activity..$^{19} \mathrm{Chu}$ et al used both the photothermal and photodynamic inactivation of cancer cells using surface plasmon resonance of Au nanoring. ${ }^{20}$

\section{Stem Cell Therapy}

Studies have established stem cell markers to cooperate a prominent role in improving the efficiency of chemotherapy and as a salient agent for targeted anticancer therapy. The efficiency of targeted cell therapy directs the need to investigate for novel biomarkers in rare orofacial cancers including oral malignancies and salivary gland malignancies and to detect the commonest malignant stem cell markers potent as indicators for premalignant lesions, progression, and the treatment of the malignant lesions. Stem cell markers are gene expression agents that are exceptionally related to stem cells. The distinctive stem cell markers are suggestive of being components of a core regulatory system in embryological mechanisms that are superintended for self-renewal, maintaining an undifferentiated state, and reorganizing adult cells. Similarly, the cancer stem cells (CSCs) present in the oral cavity cancer articulate couple of these core regulatory network proteins.

There are various techniques to isolate the CSCs and identify stemness related genes include:

- Flow cytometry

- Sphere formation-based techniques

- Side population cells ${ }^{21}$

The stemness-related markers include:

- C OCT4

- NANOG

- SOX2

Stem cells' surface markers include:

- CD44

- CD133 
- ALDH

- CD117

- CD24

- CD147

- Musahi-1

- c-MET

- BMI1

- LGR5

- Renin-angiotensin markers ${ }^{22}$

CSCs are measured as significant targets for cancer therapy. Ma et al and Benezra et al coated CSCs with polyethylene glycol (PEG). Their size (<10 nm) provided better biodistribution in a melanoma model and a prominent brightness was achieved by using NP-infused fluorescent dye. Pan et al proved that magnetic NPs are a suitable magnetic resonance imaging agent as they respond well to external magnetic fields.

Vitamin C donates electron and indulges in various biochemical activities in stem cell, synthesis of collagen, and in regulating hypoxia-inducible factor synthesis, affecting extracellular matrix remodeling and metastasis. Metered vitamin $C$ dosages inhibit glycolysis of malignant cells and wedges synthesis of nitroso compounds indicating the impending of vitamin $C$ in cancer therapy. Studies revealed vitamin $C$ to enhance cancer's immune response to anti-PD-L1 treatment through various indirect methods. ${ }^{23}$

\section{Nanotechnology-Based Radiation Immunotherapy}

Localized radiotherapeutic techniques could utilize $\alpha$ NPs in targeting tumor cells, which could help in identifying epitopes to improve immune responses. Min et al at North Carolina University reexperimented with a melanoma model with absolutely no cure. They used NPs that captured antigen to deliver tumor-specific epitopes toward antigen-presenting cells. An improved T cell response with a cure rate of $20 \%$ resulted after delivering them to the lymphocytes.

Lower growth rates were observed in the cells present in the center of the spheroid due to hypoxia (usually compensated by angiogenesis) and reduced exposure to growth factors. Tumor geometry is important to nanotechnology in delivering antigenic NPs and for local treatment effects of NPs, including radioactive AuNPor from Au NP used in hyperthermia.

\section{Ultrasound Nanotheranostics}

Ultrasound imaging and therapy are growing in demand in present times with the use of various organic nanomaterials like acoustic protein nanostructures, ${ }^{24}$ polymer-based nanomaterials, ${ }^{25}$ porphyrin- and cyanine-based dyes, ${ }^{26,27}$ and inorganic nanomaterials including carbon-based nanomaterials, ${ }^{28}$ metallic nanomaterials, ${ }^{29}$ and perfluorocarbon (PFC) nanodroplets. ${ }^{30}$ NPs enhance precision ultrasound imaging, including TME responsive ultrasound imaging, photoacoustic imaging, and phase-change imaging. It also plays a synergistic role in ultrasound therapy, in sonodynamic therapy (SDT), ${ }^{31}$ high intensity focused ultrasound (HIFU) ablation, ${ }^{32}$ and on-demand drug release controlled by ultrasound. ${ }^{33}$

Cancer treatment today includes newer, noninvasive, deeper tissue-penetration, and promising therapeutic techniques such as SDT. It activates sonosensitizers to produce huge amounts of high energy oxygen-containing molecules thereby inducing necrosis or cancer cell death under low-intensity ultrasound irradiation. ${ }^{34,35}$ The inorganic semiconductor TiO2 NPs can be efficiently used in SDT because it can penetrate deep into tissues thereby achieving a satisfactory therapeutic outcome. ${ }^{36}$

HIFU ablation efficiency is increased by using multifunctional silica nanocapsules with coencapsulated superparamagnetic magnetite NPs and liquid PFC simultaneously improved cancer diagnostic efficacy. ${ }^{37}$ Mesoporous silica materials are used for ultrasound-assisted drug delivery due to its high loading capacity, robustness, suitability for surface modification, and biocompatibility. ${ }^{38}$ Newer developments include phase-changeable, folate-targeted perfluoro hexane (PFH) nanodroplets which are infused with 10-hydroxycamptothecin (HCPT) and superparamagnetic iron oxide (Fe304) (designed as FA-HCPT-Fe304-PFP) for tumor-targeted curatives..$^{39}$ Ultrasound-enhanced immunotherapy is a promising and effective therapeutic approach for cancer. ${ }^{40}$

\section{Nanotechnology-Based Drug-Delivery Systems in Oral Cancer Therapy}

Polymer-based, lipid-based, and metal-based nanocarriers are few of the preclinical models of NPs that have been enveloped to evaluate the treatment of oral cancer. PH-sensitive PDPA (poly (2-[methacryloyloxy] ethyl phosphorylcholine) and PMPC (poly [2-diisopropylamino] ethyl methacrylate) polymersomes were used to summarize and distribute chemotherapeutic agent to the tumor cells to study the improved collective anticancer therapy. PMPC-PDPA polymersomes increase the cytotoxic effect of chemotherapeutic agents when they encapsulate Dox and paclitaxel for either individual or combined drug-delivery systems. ${ }^{41}$

Endo et al used cisplatin to evaluate the safety and usefulness of loaded polymeric nanomicelles (NC-6004) in OSCC therapeutics. In OSCC therapeutics, polymeric nanomicelles developed by conjugating Dox, and an autophagy inhibitor, LY294002 (LY) exposed that LY-loaded HPAH-DOX nanomicelles repressed tumor-cell proliferation in a synergistic manner. ${ }^{42}$ Gold-silica nanoshells infused with anti-HER2 nanobodies when applied as photothermal therapy (PTT) were found to be a potential OSCC therapeutic technique. Dox-loaded nanocarriers in the structure of silica-coated gold nanoflowers used in combination with near-infrared PTT in human tongue SCC Cal27 cells induced rapid drug release. ${ }^{43}$ DOX-methotrexate NP systems were able to decrease expression levels significantly for vascular endothelial growth factor C (VEGF-C) in addition to promoting apoptosis. Chitosan NPs loaded with cupreous complexes 
provide promising results in the in vivo mouse model of $\mathrm{KB}$ tumor. ${ }^{44}$

Natural compounds possessing proapoptotic effects like ellagic acid, curcumin, and phenolic antioxidants loaded on chitosan biopolymeric nanocarrier could be used in oral cancer therapeutics. ${ }^{45,46}$ The biodegradable polymer poly(lactic-co-glycolic acid)-PEG is utilized for creating polymeric self-assembled NPs because of its high cellular uptake and superior apoptosis properties on oral cancer cells. ${ }^{47}$ Nanodelivery of RNA could serve as a promising oral cancer therapeutic procedure.

Imai et al in his study reported that HIF1 decoy oligodeoxynucleotides caused hypoxia-mediated expression of VEGF in tumor angiogenesis. ${ }^{48}$ NPs present in the proliferating basal layer help in the local delivery of chemotherapeutic agents. ${ }^{49}$ HIF1 $\alpha$ siRNA combined to anis amide-targeted lipid-calcium-phosphate NPs and combined PDT-PTT therapy is an emerging treatment approach for oral cancer that uses rose Bengal-conjugated graphene nanoribbons specific to oral cells. ${ }^{50}$

Scientists have been targeting boron neutron-capture therapy with the use of nanocarriers for selectively building tumor-targeting compounds including boron and neutron irradiation. ${ }^{51}$ Tongue carcinoma patients were benefitted from the association of ultrasound and bubble liposome that introduced plasmid into the tongue tissue. ${ }^{52}{ }^{64} \mathrm{Cu}$ liposomes added to head and neck squamous cell carcinoma visualization have been efficient in the early diagnosis and staging of oral cancer also. ${ }^{53}$ Naringenin-loaded NPs possess an antitumor effect for the chemopreventive strategy for OSCC. Graphene NPs-coated cetuximab showed enhanced survival and reduced tumor size in an in vivo model due to prominent radiation absorption..$^{54} \mathrm{NPs}$ get accumulated in the internal organs commonly and elimination is a difficult task. ${ }^{55}$

The biological effectiveness of epigallocatechin gallate (EGCG) was improved due to induced apoptosis and reduction in angiogenesis by 10 -fold. Using EGCG was beneficial in nanochemo-prevention when used as a sustained bioactive food release..$^{56}$ Prabhu et al generated an aspirin, curcumin, and sulforaphane (ACS) combination in solid-liquid nanoparticles that were used to perform multimodal targeting of pancreatic cancer. It was observed that nanoencapsulated ACS regimens reduced tumor incidence by as high as $75 \%$ at doses 10 times lower than free drug combinations. ${ }^{57}$

In the near future, NPs-mediated drug delivery of RNAi in the diagnosis and cure of HPV as an increased risk factor for cervical or oral malignancies could prosper. Improvement in nanoencapsulation methods could enhance the RNAi release or anti-inflammatory agents. Eventually, it is our responsibility to import the best efforts in prevention and best utilize nanotechnology.

\section{Conclusion}

Nanotechnology is gaining recognition in its application in diagnostics of oral cancer. It could be used in various combinations in the therapeutics aspects of oral cancer also. The changing trends of diseases and improvements in science and technology have led the world to look toward various alternatives including nanotechnology and its increasing applicative sensitivity toward diagnosis and treatment of oral malignancies.

\section{Funding}

This study is self-funded.

\section{Conflicts of Interest}

The authors have no conflicts of interest.

\section{Acknowledgments}

None.

\section{References}

1 https://www.intechopen.com/books/biomedical-engineering-from-theory-to-applications/nanoparticles-in-biomedical-applications-and-their-safety-concerns. Accessed July 2, 2021

2 Zhang L, Gu FX, Chan JM, Wang AZ, Langer RS, Farokhzad OC. Nanoparticles in medicine: therapeutic applications and developments. Clin Pharmacol Ther 2008;83(5):761-769

3 Mudshinge SR, Deore AB, Patil S, Bhalgat CM. Nanoparticles: emerging carriers for drug delivery. Saudi Pharm J 2011;19(3):129-141

4 Cronin JG, Jones N, Thornton CA, Jenkins GJS, Doak SH, Clift MJD. Nanomaterials and innate immunity: a perspective of the current status in nanosafety. Chem Res Toxicol 2020;33(5):1061-1073

5 Chandler M, Johnson MB, Panigaj M, Afonin KA. Innate immune responses triggered by nucleic acids inspire the design of immunomodulatory nucleic acid nanoparticles (NANPs) Curr Opin Biotechnol 2020;63:8-15

6 Li Z, Chai F, Yang L, Luo X, Yang C. Mechanical properties and nanoparticles precipitation behavior of multi-component ultra high strength steel. Mater Des 2020;191:108637

7 Ferrando-Magraner E, Bellot-Arcís C, Paredes-Gallardo V, et al. Antibacterial properties of nanoparticles in dental restorative materials. A systematic review and meta-analysis. Medicina (Kaunas) 2020;56(2):55

8 Lombardo D, Kiselev MA, Caccamo MT. Smart Nanoparticles for Drug Delivery Application: Development of Versatile Nanocarrier Platforms in Biotechnology and Nanomedicine. Fratoddi I, editor. J Nanomater [Internet]. 2019;2019:3702518. Available from: https://doi.org/10.1155/2019/3702518

9 Huang J, Zhang Y, Lin Z, et al. Femtomolar detection of nucleic acid based on functionalized gold nanoparticles. Nanophotonics 2019;8(9):1495-1503

10 Chen XJ, Zhang XQ, Liu Q, Zhang J, Zhou G. Nanotechnology: a promising method for oral cancer detection and diagnosis. J Nanobiotechnology 2018;16(1):52

11 Arms L, Smith DW, Flynn J, et al. Advantages and limitations of current techniques for analyzing the biodistribution of nanoparticles. Front Pharmacol 2018;9:802

12 Inchingolo F, Santacroce L, Ballini A, Topi S, Dipalma G, Haxhirexha K, et al. Oral cancer: A historical review. Int J Environ Res Public Health. 2020;17(9):3168

13 Ghandehari S, Tabrizi MH, Ardalan P, Neamati A, Shali R. Green synthesis of silver nanoparticles using Rubia tinctorum extract and evaluation the anti-cancer properties in vitro. IET Nanobiotechnol 2019;13(3):269-274 
14 Arakeri G, Patil SG, Aljabab AS, et al. Oral submucous fibrosis: An update on pathophysiology of malignant transformation. J Oral Pathol Med 2017;46(6):413-417

15 Ceron Jayme C, Ferreira Pires A, Tedesco AC. Development of DNA polymer films as a drug delivery system for the treatment of oral cancer. Drug Deliv Transl Res 2020;10(6):1612-1625

16 Guo S, Miao L, Wang Y, Huang L. Unmodified drug used as a material to construct nanoparticles: delivery of cisplatin for enhanced anti-cancer therapy. J Control Release 2014;174:137-142

17 Zhu T, Shi L, Yu C, et al. Ferroptosis promotes photodynamic therapy: supramolecular photosensitizer-inducer nanodrug for enhanced cancer treatment. Theranostics 2019;9(11):3293-3307

18 Xue X, Huang Y, Bo R, et al. Trojan Horse nanotheranostics with dual transformability and multifunctionality for highly effective cancer treatment. Nat Commun 2018;9(1):3653

19 Gupta J, Mohapatra J, Bahadur D. Visible light driven mesoporous Ag-embedded $\mathrm{ZnO}$ nanocomposites: reactive oxygen species enhanced photocatalysis, bacterial inhibition and photodynamic therapy. Dalton Trans 2017;46(3):685-696

$20 \mathrm{Chu} \mathrm{CK}$, Tu YC, Hsiao JH, et al. Combination of photothermal and photodynamic inactivation of cancer cells through surface plasmon resonance of a gold nanoring. Nanotechnology 2016;27(11):115102

21 Ali Hosseini Rad SM, Bavarsad MS, Arefian E, Jaseb K, Shahjahani M, Saki N. The role of microRNAs in stemness of cancer stem cells. Oncol Rev 2013;7(1):e8

$22 \mathrm{Fu} \mathrm{J}, \mathrm{Wu} \mathrm{Z}$, Liu J, Wu T. Vitamin C: a stem cell promoter in cancer metastasis and immunotherapy. Biomed Pharmacother 2020;131:110588

23 Michel T, Fehrenbach J, Lobjois V, et al. Mathematical modeling of the proliferation gradient in multicellular tumor spheroids. J Theor Biol 2018;458:133-147

24 Lanza GM, Winter PM, Caruthers SD, et al. Theragnostics for tumor and plaque angiogenesis with perfluorocarbon nanoemulsions. Angiogenesis 2010;13(2):189-202

25 Li W, Chen X. Gold nanoparticles for photoacoustic imaging. Nanomedicine (Lond) 2015;10(2):299-320

26 Gong H, Peng R, Liu Z. Carbon nanotubes for biomedical imaging: the recent advances. Adv Drug Deliv Rev 2013;65(15):1951-1963

27 Li J, Rao J, Pu K. Recent progress on semiconducting polymer nanoparticles for molecular imaging and cancer phototherapy. Biomaterials 2018;155:217-235

28 Sheng $\mathrm{Z}, \mathrm{Hu} \mathrm{D}$, Zheng $\mathrm{M}$, et al. Smart human serum albumin-indocyanine green nanoparticles generated by programmed assembly for dual-modal imaging-guided cancer synergistic phototherapy. ACS Nano 2014;8(12):12310-12322

29 Shapiro MG, Goodwill PW, Neogy A, et al. Biogenic gas nanostructures as ultrasonic molecular reporters. Nat Nanotechnol 2014;9(4):311-316

30 Huang P, Qian X, Chen Y, et al. Metalloporphyrin-encapsulated biodegradable nanosystems for highly efficient magnetic resonance imaging-guided sonodynamic cancer therapy. J Am Chem Soc 2017;139(3):1275-1284

31 Wang X, Chen H, Chen Y, et al. Perfluorohexane-encapsulated mesoporous silica nanocapsules as enhancement agents for highly efficient high intensity focused ultrasound (HIFU). Adv Mater 2012;24(6):785-791

32 Moon GD, Choi SW, Cai X, et al. A new theranostic system based on gold nanocages and phase-change materials with unique features for photoacoustic imaging and controlled release. J Am Chem Soc 2011;133(13):4762-4765

33 Pan X, Bai L, Wang H, et al. Metal-organic-framework-derived carbon nanostructure augmented sonodynamic cancer therapy. Adv Mater 2018;30(23):e1800180
34 Jing Zhu CC, Li D, Pang X, et al. En Ren, Jingliang Cheng, Xiaoyuan Chen, and Gang Liu*, Fe(III)-Porphyrin Sonotheranostics: A Green TripleRegulated ROS Generation Nanoplatform for Enhanced Cancer Imaging and Therapy. Adv Funct Mater 2019;1904056

35 Wang X, Wang W, Yu L, Tang Y, Cao J, Chen Y. Site-specific sonocatalytic tumor suppression by chemically engineered single-crystalline mesoporous titanium dioxide sonosensitizers. J Mater Chem B Mater Biol Med 2017;5(24):4579-4586

36 Niu D, Wang X, Li Y, et al. Facile synthesis of magnetite/perfluorocarbon co-loaded organic/inorganic hybrid vesicles for dual-modality ultrasound/magnetic resonance imaging and imaging-guided high-intensity focused ultrasound ablation. Adv Mater 2013;25(19):2686-2692

37 Manzano M, Vallet-Regí M. Ultrasound responsive mesoporous silica nanoparticles for biomedical applications. Chem Commun (Camb) 2019;55(19):2731-2740

38 Liu J, Xu F, Huang J, et al. Low-intensity focused ultrasound (LIFU)-activated nanodroplets as a theranostic agent for noninvasive cancer molecular imaging and drug delivery. Biomater Sci 2018;6(11):2838-2849

39 Pang X, Liu X, Cheng Y, et al. Sono-immunotherapeutic nanocapturer to combat multidrug-resistant bacterial infections. Adv Mater 2019;31(35):e1902530

40 Rao L, Meng QF, Bu LL, et al. Erythrocyte membrane-coated upconversion nanoparticles with minimal protein adsorption for enhanced tumor imaging. ACS Appl Mater Interfaces 2017;9(3):2159-2168

41 Endo K, Ueno T, Kondo S, et al. Tumor-targeted chemotherapy with the nanopolymer-based drug NC-6004 for oral squamous cell carcinoma. Cancer Sci 2013;104(3):369-374

42 Fekrazad R, Hakimiha N, Farokhi E, et al. Treatment of oral squamous cell carcinoma using anti-HER2 immunonanoshells. Int J Nanomedicine 2011;6:2749-2755

43 Abbasi MM, Monfaredan A, Hamishehkar H. Seidi K, JahanbanEsfahlan R. Novel DOX-MTX nanoparticles improve oral SCC clinical outcome by down regulation of lymph dissemination factor VEGF-C expression in vivo: oral and IV modalities. Asian Pac J Cancer Prev 2014;15(15):6227-6232

44 Lin M, Wang D, Liu S, et al. Cupreous complex-loaded chitosan nanoparticles for photothermal therapy and chemotherapy of oral epithelial carcinoma. ACS Appl Mater Interfaces 2015;7(37):20801-20812

45 Mazzarino L, Loch-Neckel G, Bubniak LdosS, et al. Curcumin-loaded chitosan-coated nanoparticles as a new approach for the local treatment of oral cavity cancer. J Nanosci Nanotechnol 2015;15(1):781-791

46 Arulmozhi V, Pandian K, Mirunalini S. Ellagic acid encapsulated chitosan nanoparticles for drug delivery system in human oral cancer cell line (KB) Colloids Surf B Biointerfaces 2013;110:313-320

47 Wang ZQ, Liu K, Huo ZJ, et al. A cell-targeted chemotherapeutic nanomedicine strategy for oral squamous cell carcinoma therapy. J Nanobiotechnology 2015;13:63

48 Dong G, Lin XH, Liu HH, et al. Intermittent hypoxia alleviates increased VEGF and pro-angiogenic potential in liver cancer cells. Oncol Lett 2019;18(2):1831-1839

49 Bharathiraja S, Manivasagan P, Santha Moorthy M, et al. Photo-based PDT/PTT dual model killing and imaging of cancer cells using phycocyanin-polypyrrole nanoparticles. Eur J Pharm Biopharm 2018;123:20-30

50 Yuan TZ, Xie SQ Qian CN. Boron neutron capture therapy of cancer: critical issues and future prospects. Thorac Cancer 2019;10(12):2195-2199

51 Sugano M, Negishi Y, Endo-Takahashi Y, et al. Gene delivery system involving Bubble liposomes and ultrasound for the efficient in vivo delivery of genes into mouse tongue tissue. Int J Pharm 2012;422(1-2):332-337 
52 Mahakian LM, Farwell DG, Zhang $\mathrm{H}$, et al. Comparison of PET imaging with 64Cu-liposomes and 18F-FDG in the 7,12-dimethylbenz[a]anthracene (DMBA)-induced hamster buccal pouch model of oral dysplasia and squamous cell carcinoma. Mol Imaging Biol 2014;16(2):284-292

53 Fury MG, Sherman EJ, Rao SS, et al. Phase I study of weekly nab-paclitaxel + weekly cetuximab + intensity-modulated radiation therapy (IMRT) in patients with stage III-IVB head and neck squamous cell carcinoma (HNSCC). Ann Oncol 2014;25(3):689-694

54 Gherman C, Tudor MC, Constantin B, et al. Pharmacokinetics evaluation of carbon nanotubes using FTIR analysis and histological analysis. J Nanosci Nanotechnol 2015;15(4):2865-2869
55 Siddiqui IA, Adhami VM, Ahmad N, Mukhtar $\mathrm{H}$. Nanochemoprevention: sustained release of bioactive food components for cancer prevention. Nutr Cancer 2010;62(7):883-890

56 Grandhi BK, Thakkar A, Wang J, Prabhu S. A novel combinatorial nanotechnology-based oral chemopreventive regimen demonstrates significant suppression of pancreatic cancer neoplastic lesions. Cancer Prev Res (Phila) 2013;6(10):1015-1025

57 Schmalz G, Hickel R, van Landuyt KL, Reichl FX. Scientific update on nanoparticles in dentistry. Int Dent J 2018;68(5):299-305 